\title{
The role of echocardiography in diagnosing carditis in the setting of acute rheumatic fever
}

The letter was shown to Professor Vijayalakshmi and her colleagues, who replied as follows:

Dear Sir,

We thank Alessandra Benettoni and her colleagues for their endorsement of our emphasis on the importance of performing echocardiography routinely in all patients suspected of having rheumatic fever, and our suggestion that echocardiographic assessment should be included as a major criterion of rheumatic fever. Our scoring system, which we constructed meticulously on the basis of our considerable previous experience, included the use of colour Doppler interrogation to measure the regurgitant jet, as recommended by the American Society of Echocardiography (Reference 12 in our article ${ }^{1}$ ). Thus, this feature is used, along with the findings of thickened valvar leaflets with reduced mobility, mitral valvar prolapse, a beaded appearance to the valvar components, hyperechogenicty of the submitral structures, pericardial effusion, and cordal tears as additional supportive findings, to ensure that the diagnosis of carditis or valvitis is more accurate. Our supporting video showed such significant mitral regurgitation, along with thickened aortic and mural leaflets of the mitral valve supported by rolled-up and torn tendinous cords, these features combining to produce a score of 6 - see www.journals.cambridge.ort/ jid_CTY.

Our approach in using all the other features of the valves, along with colour Doppler interrogation to measure the regurgitant jet, prevents both overand under-diagnosis. In our opinion, such a system of scoring is easily achieved and feasible anywhere with any equipment. Once aware of these specific features, the investigator can easily apply the system in clinical routine practice. Indeed, we believe that any physician investigating a patient suspected to have acute rheumatic fever can easily appreciate all the 8 features that we have highlighted in our system of scoring. Furthermore, having recognised what needs to be looked for, the features can readily be recognised by any experienced echocardiographic technician. What the mind does not know, the eyes will not see! We very much agree with their observation that the patients presenting with chorea are more prone to have carditis. In our pilot study, ${ }^{2}$ over two-thirds of our patients with rheumatic chorea had echocardiographic evidence of cardiac involvement in the absence of any other criterion!

Ishwarappa B. Vijayalakshmi Professor of Pediatric Cardiology Sri Jayadeva Institute of Cardiology Bangalore, Karnataka, India

Tel: 91802330 2031, mobile: 094484940984 ; Fax: 91802297 7236; E-mail:dr_vj@botmail.com

\section{References}

1. Vijayalakshmi IB, Vishnuprabhu OR, Chitra N, Rajasri R, Anuradha TV. The efficacy of echocardiographic criterions for the diagnosis of carditis in acute rheumatic fever. Cardiol Young 2008; 18: 586-592.

2. Vijayalakshmi IB, Mithravinda J, Prabhu Deva AN. Role of Echocardiography in diagnosing carditis in the setting of acute rheumatic fever. Cardiol Young 2005; 15: 583-588. 